Société d'histoire de la révolution de 1848 et des

révolutions du XIXe siècle

$34 \mid 2007$

La bourgeoisie : mythes, identités et pratiques

\title{
Construire et déconstruire la bourgeoisie : discours politique et imaginaire social au début du XIX siècle
}

Constructing and deconstructing bourgeoisie. Political discourse and social imaginary in post-revolutionary France

Sarah Maza

\section{(2) OpenEdition}

Journals

Édition électronique

URL : http://journals.openedition.org/rh19/1262

DOI : $10.4000 /$ rh19.1262

ISSN : $1777-5329$

Éditeur

La Société de 1848

Édition imprimée

Date de publication : 1 juin 2007

Pagination : 21-37

ISSN : $1265-1354$

\section{Référence électronique}

Sarah Maza, "Construire et déconstruire la bourgeoisie : discours politique et imaginaire social au début du XIXe siècle », Revue d'histoire du XIXe siècle [En ligne], 34 | 2007, mis en ligne le 01 juin 2009, consulté le 01 mai 2019. URL : http://journals.openedition.org/rh19/1262 ; DOI : 10.4000/rh19.1262 


\section{SARAH MAZA}

\section{Construire et déconstruire la bourgeoisie : discours politique et imaginaire social au début du XIX'e siècle}

On connait la synthèse classique des livres d'histoire : la bourgeoisie moderne naît au sein de la société féodale, se développe au XVIII ${ }^{e}$ siècle, accède au pouvoir politique avec l'explosion révolutionnaire, prélude à son hégémonie sociale et culturelle au XIX ${ }^{\mathrm{e}}$ siècle ${ }^{1}$. Mais on sait aussi les problèmes que pose ce scénario traditionnel. Depuis maintenant plusieurs décennies, historiens et spécialistes de l'histoire économique soulignent la place très marginale de tout capitalisme de type moderne dans l'économie du siècle des Lumières. Une lecture différente de la Révolution a mis l'accent, depuis les années 1980, sur les transformations idéologiques et politiques qu'elle a engendrées, soulignant les continuités socio-économiques entre l'avant et l'après $1789^{2}$. Même les historiens du XIX siècle s'accordent pour souligner qu'on ne peut parler de concentration capitaliste avant le Second Empire au plus tôt - la France reste avant le milieu du XIX ${ }^{e}$ siècle un pays fortement agraire dont même les industries de pointe, tel le textile, demeurent enracinées dans un contexte rural et affichent des caractéristiques au plus proto-capitalistes ${ }^{3}$.

Que signifie alors parler d'avènement ou d'hégémonie bourgeoise en dehors d'un changement net de régime économique? On répondra, comme le font la plupart des historiens récents, que le concept de bourgeoisie aux $\mathrm{XVIII}^{\mathrm{e}}$ et $\mathrm{XIX}^{\mathrm{e}}$ siècles doit se comprendre de façon plus souple que dans la vulgate marxiste, comme désignant les nantis d'une société post-aristocratique, depuis les notables et les grands propriétaires terriens jusqu'aux employés et aux commerçants que leur position met à l'abri du besoin et

1. Cet article reprend certaines des conclusions de mon livre paru récemment en anglais. Pour plus de détails voir Sarah Maza, The Myth of the French Bourgeoisie : An Essay on the Social Imaginary 17501850, Cambridge et Londres, Harvard University Press, 2003.

2. Voir par exemple Patrick O'Brien et Caglar Keydar, Economic Growth in Britain and France, 1780-1914: Two Paths to the Twentieth Century, Londres, Allen and Unwin, 1978; Colin Heywood, The Development of the French Economy, Cambridge, Cambridge University Press, 1992. Pour la relecture de la révolution voir, outre les travaux bien connus de François Furet et Lynn Hunt, la synthèse de William Doyle, Origins of the French Revolution Oxford, Oxford University Press, 1980. 1992.

3. Voir par exemple Peter McPhee, A Social History of France, 1780-1880, Londres, Routledge, 
protège de l'ignominie du travail manuel ${ }^{4}$. On s'accorde donc aujourd'hui pour désigner la bourgeoisie comme la classe dominante de la France postrévolutionnaire, tout en reconnaissant d'emblée que le lien entre bourgeoisie et capitalisme ne concerne qu'une partie, sans doute très minoritaire, du groupe ainsi désigné. Il est aussi entendu, comme l'ont souligné nombre de travaux donc ceux, très importants, d'Adeline Daumard, que la bourgeoisie dans ce sens comprend un large éventail de groupes de statut et fortune très divers, depuis le petit commerçant ou le maitre artisan jusqu'au richissime banquier en passant par un grand nombre d'occupations différentes dans les secteurs tant public que privé 5 .

Et c'est là l'un des problèmes que soulève la définition de la bourgeoisie dans la France moderne et contemporaine. Si l'on s'accorde pour détacher «bourgeoisie» de "capitalisme», si l'on concède que ce n'est pas le rapport à un mode de production qui définit ce groupe, pourquoi donc s'acharner à loger à la même enseigne un Rothschild - voire un Thiers ou Guizot - et un petit épicier de province? Que donc auraient ces gens en commun? On entend parfois que la bourgeoisie se définit par des éléments de culture, des pratiques communes telles que l'emploi des domestiques, le culte de la vie familiale et de la femme au foyer, ou certaines normes d'éducation, d'hygiène et de propriété. L'approche est sans doute prometteuse encore que, malgré certaines brillantes exceptions, on dispose de relativement peu d'études approfondies des cultures bourgeoises dans la période 1750-1850, trop peu certainement pour établir une synthèse à l'heure qu'il est. Trop souvent, encore, on a tendance à partir du principe que la culture bourgeoise est une donnée que l'on n'a pas à expliquer car tout un chacun sait ce que c'est : le piano au salon, l'église le dimanche, les convenances affichées et l'adultère caché.

Outre la question de la définition "objective» d'un groupe extrêmement hétéroclite, un autre problème se pose qui est celui du refus d'affirmation identitaire. Car si c'est un cliché que de faire remarquer que dans le contexte français le bourgeois ce n'est jamais soi, c'est toujours l'autre, il n'en reste pas moins vrai que cette observation soulève une réalité, et une problématique historique, cruciales. En contraste frappant avec le monde anglo-américain où depuis le XVIII e siècle tout un chacun se réclame de la middle class, on note l'absence dans le contexte français - au moins avant 1850 - d'un corpus important, soutenu, de témoignages ou d'écrits de groupes se disant bourgeois et fiers de l'être ${ }^{6}$ (la seule exception majeure sera considérée plus avant). Sans doute trouve-t-on des témoignages épars, mais en quantité très

4. Voir par exemple William H. Sewell, A Rhetoric of Bourgeois Revolution: The Abbé Sieyès and What is the Third Estate?, Durham (États-Unis), Duke University Press, 1994.

5. Elinor Barber, The Bourgeoisie in Eighteenth-Century France, Princeton, Princeton University Press, 1955; Régine Pernoud, Histoire de la bourgeoisie en France, Paris, Le Seuil, 1960-1962; Adeline Daumard, Les Bourgeois et la bourgeoisie en France depuis 1815, Paris, Aubier, 1987.

6. Pour les États-Unis voir Benjamin DeMott, The Imperial Middle: Why Americans Can't Think 
faible comparée non seulement à l'équivalent anglo-américain, mais aussi à la masse énorme d'écrits qui depuis l'Ancien Régime ridiculisent et dénoncent la bourgeoisie. Au niveau du discours, l'identité bourgeoise ne semble exister que par la négative. Or, peut-on dire qu'un groupe existe si ses membres ne s'en réclament jamais?

D'où l'hypothèse délibérément provocatrice avancée dans l'ouvrage que nous avons publié récemment : pour la période 1750-1850, du moins, la bourgeoisie est un mythe plutôt qu'un groupe social. Il ne s'agit pas, évidemment, de mettre en doute l'existence, dans toute cette période, de larges groupes sociaux de statut moyen et de résidence surtout - mais non uniquement - urbaine. Mais cette hypothèse est avancée dans l'espoir de stimuler le débat par la provocation, au moyen d'une remise en cause radicale de l'acquis. Peut-on contourner le problème sociologique en essayant de cerner la bourgeoisie par le biais des discours et des représentations? Y a-t-il un discours du bourgeois sur lui-même, et sinon comment expliquer cette absence? Si le bourgeois ne se définit pas lui-même, qui le définit, et pourquoi?

Dans la discussion qui suit, nous mettrons l'accent, dans le cadre d'un argument plus large, sur l'exception principale à la règle ci-dessus, à ce silence de la bourgeoisie sur elle-même : le discours politico-historique des libéraux de la Restauration. La génération des Thierry, Guizot, Royer-Collard ou Mignet produit, on le sait, un récit triomphaliste au sein duquel le bourgeois fait figure de héros tant pour le passé que pour le présent et l'avenir. Nous nous interrogerons sur les raisons de cette tentative de construire ainsi le social par le politique - le «juste milieu» politique s'incarnant dans une "classe moyenne»- et plus encore sur les raisons de son insuccès. Pourquoi cette tentative d'ériger, sur le modèle anglais, une middle class comme clé de voûte de la culture politique française s'est-elle soldée par un échec? Comment comprendre cet épisode au sein d'une histoire de l'imaginaire social français où «la bourgeoisie» fait surtout figure de repoussoir?

Il convient d'abord, afin d'établir l'originalité du discours triomphaliste sur la bourgeoisie entre 1815 et 1830 , d'effectuer un rapide survol de la place de la bourgeoisie dans l'imaginaire social du siècle précédent, aussi bien à la fin de l'Ancien Régime que sous la Révolution. Deux faits majeurs se dégagent. D'une part, et l'on s'y attend, les perceptions de la bourgeoisie au XVIII siècle sont marquées pour le moins d'une forte ambivalence, voire le plus souvent d'hostilité et de mépris. Mais de façon plus importante, ce qui frappe c'est l'absence de référence à une telle classe alors même que les circonstances sembleraient s'y prêter : dans le commentaire sur les boule- 
versements socio-économiques du Siècle des Lumières, une telle classe n'est presque jamais nommée, pas plus qu'elle ne l'est dans la discussion de l'immense lame de fond politique de 1789 et après.

Il existe, bien sûr, une définition neutre de la bourgeoisie sous l'Ancien Régime. "La bourgeoisie» a existé depuis des siècles en tant que catégorie urbaine au statut juridique précis - on est bourgeois comme on est notaire ou orfevre. De par sa position sociale prééminente, ses avantages fiscaux, l'oisiveté de jure ou de facto de ses membres - le plus souvent rentiers - la «bourgeoisie d'Ancien Régime» fait figure de sous-aristocratie ${ }^{7}$. Et c'est justement l'un des problèmes qu'a toujours posés ce groupe : à la différence de la notion anglo-américaine de middle class, l'idée française de bourgeoisie a toujours été entachée de connotations de privilège. Mais aussi d'une forte dose d'ambivalence : le discours sur le bourgeois est dès le départ un discours d'altérité largement hostile. Les dictionnaires de l'Ancien Régime, ceux de l'Académie française, de Furetière, de Trévoux et de Richelet, définissent le bourgeois d'abord en termes techniques ("Citoyen, habitant d'une ville»), pour ensuite le cerner au moyen d'un double rejet ${ }^{8}$.

Pour l'ouvrier ou le domestique, notent les dictionnaires, le «bourgeois" c'est le "patron" : "On doit servir le bourgeois, on ne doit pas tromper le bourgeois". Pour l'homme du monde ou de la cour, le bourgeois, c'est aussi l'autre : un être mal dégrossi, "pas assez poli, trop familier", qui manque de manières, de galanterie ou d'esprit. "Cela sent bien son bourgeois", dit-on. $\mathrm{Au}$ XVIII siècle, il existe une tradition pluriséculaire, dont Monsieur Jourdain n'est que le représentant le mieux connu, de satire antibourgeoise 9 . La bourgeoisie, zone traditionnelle de transition sociale entre la roture et la noblesse, inquiète par sa position liminaire au sein d'une société fortement hiérarchisée. Le bourgeois est un monstre parce que c'est un métis. C'est du moins ce que l'on peut lire entre les lignes d'un commentaire dédaigneux de Marivaux en 1717 : «Le bourgeois de Paris, Madame, est un animal mixte, qui tient du grand seigneur et du peuple. Quand il a de la noblesse dans ses manières, il est presque toujours singe; quand il a de la petitesse, il est naturel. Ainsi il est noble par imitation, et peuple par disposition. ${ }^{10}$

Le plus frappant, cependant, c'est l'absence de la bourgeoisie dans les grands débats sociaux du XVIII siècle. Ce n'est pas autour de la "bourgeoisie» que déferlent commentaires ou débats. Le groupe social qui fait

7. Régine Pernoud, Histoire de la bourgeoisie, volume 1, chapitres 2 et 3; Michel Vovelle et Daniel Roche, "Bourgeois, rentiers, propriétaires : éléments pour la définition d'une catégorie sociale à la fin du XVIII" siècle", Actes du $84^{e}$ Congrès des sociétés savantes, Dijon, 1959, Paris, Imprimerie nationale, 1960 , p. 419-452; Joseph Di Corcia, "Bourg, Bourgeois, Bourgeois de Paris from the Eleventh to the Eighteenth Century", dans Journal of Modern History, $\mathrm{n}^{\circ}$ 50, juin 1978, p. 207-231.

8. La discussion qui suit s'appuie sur les articles «bourgeois» et "bourgeoisie» dans les dictionnaires Richelet de 1680, Furetière pour 1690, de Trévoux pour 1704, de l'Académie française pour 1694, 1754 , et 1798 .

9. Jean Alter, Les origines de la satire antibourgeoise en France, 2 volumes, Genève, Droz, 1970.

10. Pierre Carlet de Marivaux, Journaux et ouvres diverses, Paris, Garnier, 1969, p. 14. 
couler l'encre, avant comme pendant la Révolution, c'est la noblesse. Celleci est l'enjeu majeur des débats historiques de l'époque, par exemple, des affrontements entre historiens pro-nobles, tels le comte de Boulainvilliers, et leurs antagonistes pro-monarchistes, tels l'abbé Dubos. La noblesse est-elle à l'origine de la nation, antérieure à la monarchie, le roi un primus inter pares? Ou bien se trouvait-elle dès l'origine soumise au roi, une caste de serviteurs parfois rebelles ${ }^{11}$ ? Chez les historiens progressistes comme Mably, on a beau jeu de dénoncer la violence aristocratique et "l'anarchie féodale», personne ne songe à designer la bourgeoisie pour succéder aux nobles ${ }^{12}$.

De même, le débat social qui, au milieu du siècle, enflamme les esprits et tarit les encriers, c'est encore une polémique autour de la noblesse. Des douzaines de pamphlets relèvent le défi de l'abbé Coyer; dans un ouvrage de 1756 qui fait immédiatement scandale, celui-ci avance l'argument que, pour le bien de la nation, les nobles devraient pouvoir s'adonner au commerce sans déroger. Les commerçants et autres roturiers? Personne ne semble s'inquiéter de leur honneur, de leurs droits, de leurs devoirs ou de leur histoire, tant il est vrai qu'au sein des élites, c'est la noblesse, et elle seule, dont le statut définit symboliquement celui de la nation.

L'«invisibilité» du bourgeois dans les grands débats sociaux du siècle est frappante, alors même que l'implication des classes moyennes urbaines dans certains grands changements nous semble aujourd'hui évidente. L'essor extraordinaire du commerce et de la consommation urbaine au Siècle des Lumières ainsi que l'accès à un certain confort pour des catégories moyennes et modestes de la population sembleraient clairement se ranger sous la rubrique de l'avènement bourgeois. Mais les contemporains ne comprennent pas ainsi la nouvelle abondance du vêtement et du meuble, le confort accru des intérieurs, l'essor de biens matériels, de la montre aux médicaments, que peut s'offrir tout un chacun à partir d'une modeste aisance ${ }^{13}$. Pour les commentateurs, qu'ils soient physiocrates, moralistes chrétiens, ou adeptes de Rousseau, les angoisses créées par la mobilité sociale accrue et la nouvelle prolifération des biens de consommation se traduisent par la dénonciation du «luxe», thème de plusieurs centaines de traités et de pamphlets. Pour ne citer qu'une jérémiade typique parmi des douzaines d'autres, similaires : "Quelle philosophie que celle dont la mollesse et les plaisirs sont la base, où la distinction des rangs, des états, des conditions est aussi oubliée que la délicatesse des

11. Jean-Marie Goulemot, Le Règne de l'histoire : Discours historiques et révolutions, XVII'-XVIII siècles, Paris, Albin Michel, 1996; Marie-France Piguet, Classe. Histoire du mot et genèse du concept, Lyon, Presses Universitaires de Lyon, 1996.

12. Johnson Kent Wright, A Classical Republican in Eighteenth-Century France : The Political Thought of Mably, Stanford, Stanford University Press, 1997; voir surtout le chapitre 6.

13. Annick Pardailhé-Galabrun, La Naissance de l'intime : 3000 foyers parisiens aux XVII $-X V I I I^{e}$ siècles, Paris, Presses Universitaires de France, 1988. Daniel Roche, Histoire des choses banales. Naissance de la consommation, XVII'-XVIII siècles, Paris, Fayard, 1993; Colin Jones, "The Great Chain of Buying: Medical Advertisements, the Bourgeois Public Sphere, and the Origins of the French Revolution", American Historical Review, ${ }^{\circ} 101,1996$, p. 13-40. 
sentiments, la probité, et l'intérêt de la patrie! ${ }^{14}$ La dénonciation du «luxe» ne concerne pas spécialement une bourgeoisie ou une classe moyenne, mais bien l'ensemble d'une société corrompue par la contamination en cascade de nouvelles valeurs matérialistes.

Mais si «la bourgeoisie» en tant que telle n'est pas mise en évidence dans les grands débats sociaux de la fin de l'Ancien Régime, elle n'est pas plus présente, en nom du moins, dans le discours de la Révolution elle-même. Parlet-on de «bourgeoisie» sous la Révolution? Pas explicitement, à l'ordinaire, et certainement pas en bien. Dès le début il est beaucoup question, bien sûr, du tiers-état. Mais celui-ci est toujours compris, c'est là son essence même, comme une catégorie universelle comprenant aussi bien les travailleurs les plus pauvres que les riches roturiers. Comme l'écrit Sieyès lui-même, «le tiers-état est une nation complète ${ }^{15}$. Qu'on se rappelle seulement les classiques images représentant les trois ordres : le tiers y est le plus souvent figuré sous les traits d'un paysan en haillons ${ }^{16}$. Les représentations écrites par Sieyès et ses nombreux collègues confirment que le tiers n'est nullement assimilé à une bourgeoisie ou classe moyenne, mais bien identifié à l'ensemble de la population productive. Ce n'est donc pas, au moins explicitement, dans le concept de tiers-état que l'on trouvera l'image d'une bourgeoisie en tant que telle.

L'argument selon lequel le tiers-état se confond avec la nation entière - parasites sociaux en moins -, et de ce fait représente "la Nation», a de profondes conséquences en ce que le débat autour des États généraux lègue au pays - ou du moins à la tradition républicaine - une vision résolument universaliste de la société. C'est ce qu'exprime en négative l'obsession de "l'aristocrate", l'ennemi qui en refusant la Révolution récuse par là même le consensus social. Les interprétations traditionnelles de la Révolution voient dans le discours anti-aristocratique l'expression «en creux» d'une conscience de classe bourgeoise. Or «bourgeois» n'est pas le seul contraire possible d'aristocrate; puisque l'aristocrate est celui qui refuse l'inclusion dans le groupe, son opposé plus vraisemblable c'est "peuple» ou «nation». En tout cas, le débat social des débuts de la Révolution a pour conséquence de définir le peuple français comme indivisible. Un groupe social qui se démarque de la nation, à plus forte raison un groupe aisé ou puissant, sera dorénavant taxé d'"aristocratie». C'est là, et cela le restera longtemps, un obstacle majeur à l'auto-désignation d'une classe bourgeoise.

Cela explique aussi que dans les rares instances où, sous la Révolution, la bourgeoisie est nommée, elle est typiquement honnie. La bourgeoisie légale

14. Béliard, Lettres critiques sur le luxe, Amsterdam, 1771, p. 37-38.

15. Titre du premier chapitre de Qu'est-ce que le tiers-état?

16. Voir par exemple l'iconographie dans French Caricature and the French Revolution, 1789-1799, Los Angeles, University of California Press, 1988, images n $27,28,30,31,33,34,36,40,41,42$, 43. 
est vite abolie, comme le sont tous les groupes détenteurs de privilèges. Les patriotes les plus nantis distinguent, dans leurs écrits privés, les "gens de bien" ou les "honnêtes gens» de la racaille révolutionnaire inculte et violente ${ }^{17}$. Mais il n'est jamais question d'admettre en public ou officiellement une telle distinction. La bourgeoisie est rarement présente dans le discours révolutionnaire, et quand elle l'est, c'est dans un contexte peu flatteur. Dans telle section populaire en 1793, par exemple, on dénonce «l'aristocratie prêtre, noble, parlementaire et bourgeoise ${ }^{18}$. Dans le registre des ennemis des sans-culottes, «l'aristocratie bourgeoise» figure en bonne place parmi les reliques détestées de l'Ancien Régime.

Robespierre lui-même emploie indifféremment les expressions « aristocratie bourgeoise» ou «bourgeoisie aristocratique» quand il égrène la litanie des ennemis de la Révolution ${ }^{19}$. Il est évident que pauvres et nantis au sein de la Révolution ont eu dès le départ, en dépit de l'idéologie officielle d'harmonie sociale, des intérêts et des expériences radicalement différents, et que cette différence ne fera que s'accroître au fil des ans comme en témoignent des épisodes tels que les journées de germinal et prairial an III ou la Conjuration des Égaux. Il n'en reste pas moins vrai, cependant, qu'on ne verra apparaître ni à la fin du XVIIIe siècle, ni à l'aube du suivant, aucun discours d'autoidentification bourgeoise. Sous la Révolution, encore bien plus qu'avant, le bourgeois c'est l'autre.

Méprisée sous l'Ancien Régime, honnie mais surtout gommée sous la révolution, «la bourgeoisie» n'est jamais, il s'en faut, invoquée comme norme sociale - alors même que, s'il faut encore le souligner, une proportion large et grandissante de la population correspond à ce que nous-même serions tentés d'appeler une bourgeoisie. C'est cela qui rend d'autant plus soudaine et plus exceptionnelle l'«invention» de la bourgeoisie dans le discours historique et politique de la Restauration.

On serait tenté d'écrire que la bourgeoisie naît d'un coup de poignard asséné dans la nuit du 13 février 1820. (Le poignard en question, celui de l'assassin Louvel, perce le cœur du duc de Berry, déclenchant dans les sphères gouvernementales un raz-de-marée ultra-aristocratique et réactionnaire.) Une telle provocation n'aurait pour but que de souligner à quel point l'invocation explicite, la glorification même, d'une identité bourgeoise sous la Restauration est étroitement liée au contexte politique de l'époque.

17. On peut suivre cette évolution dans Charles-Élie, marquis de Ferrières, Correspondance inédite, Paris, 1932, et Adrien Duquesnoy, Journal d'Adrien Duquesnoy, député du tiers-état de Bar-le-Duc, Paris, Alphonse Picard et fils, 1894, 2 volumes.

18. Walter Markov et Albert Soboul, Die Sansculotten von Paris : Dokumente zur Geschichte der Volksbewegung, 1793-1794, Berlin, Akademie Verlag, 1957, p. 248.

19. Par exemple, Maximilien de Robespierre, Euvres, New York, Burke Franklin, 1970, 3 volumes, volume 1, p. 230-240, 283, 326-327, 426-427. 
C'est bien sûr dans la dynamique politique plus large de la France postimpériale que l'on doit chercher l'explication de l'apparition soudaine d'un discours pro-bourgeois. Car, dans les années qui suivent 1815, on ne voit pas plus qu'avant de changement qualitatif dans les structures socio-économiques de la nation. La croissance de l'économie dans ces années se maintient autour de 2 à $3 \%$ par an. Certes, les plus grandes villes, la capitale surtout, continuent l'accroissement galopant qu'elles ont entamé au siècle précédent ${ }^{20}$ Le succès que rencontre la figure du héros bourgeois tel que le présentent les historiens de la génération de Guizot doit sans doute beaucoup à l'extension et au renforcement de réseaux gouvernants à l'échelle municipale. Encore faut-il noter que, comme le souligne l'ouvrage important de David Garrioch, la naissance d'une "bourgeoisie parisienne" post-révolutionnaire à l'échelle de la ville entière et non du quartier doit beaucoup plus au rôle de l'État (éducation d'élite unifiée, postes administratifs) qu'aux transformations industrielles ou commerciales ${ }^{21}$.

Mais si l'on veut poser l'hypothèse d'une genèse «politique» de la bourgeoisie, il convient, on l'a dit, de se tourner vers la dynamique politico-idéologique de la Restauration. La période après 1815 a ceci de particulier que pour la première fois un groupe social, la noblesse, tente de dominer l'arène politique en se réclamant de son identité de classe historique. Du coup, leurs adversaires politiques forgent en les contrecarrant leur propre récit historique. Il serait excessif d'avancer que «la bourgeoisie» fut une pure invention politique, bien entendu. Mais étant donné certaines transformations introduisant inéluctablement la modernité au long terme - transports, commerce, urbanisation, ouverture progressive de la vie politique - c'est le combat dans l'arène politique de la Restauration qui a le plus contribué à l'identification, un temps positive, d'une «bourgeoisie» au rôle central dans l'histoire de la nation.

Les pères de ce nouveau discours sur la bourgeoisie sont les membres du groupe bien connu de politiciens et d'écrivains libéraux de la Restauration, ceux qui forment le noyau de l'opposition politique aux ultras : Thierry, Guizot, Royer-Collard, Barante, Thiers, Mignet, et quelques autres. C'est aussi bien en tant qu'acteurs dans la vie politique du régime que de journalistes et professeurs qu'ils ont marqué de leur empreinte l'imaginaire social de la nation ${ }^{22}$.

L'un des enjeux majeurs du combat politique sous Louis XVIII a été, on le sait, l'extension du droit de vote. Tout en partageant une perspective fortement élitiste, ultras et libéraux s'affrontent autour de la question des critères qui

20. Peter McPhee, A Social History of France, pp. 131-133; William H. Sewell, Work and Revolution in France: The Language of Labour from the Old Regime to 1848, Cambridge, Cambridge University Press, 1980, p. 146-153.

21. David Garrioch, The Formation of the Parisian Bourgeoisie, 1690-1830, Cambridge, Harvard University Press, 1996.

22. Pour l'histoire de ce groupe on peut consulter, Alan Spitzer, The French Generation of 1820, Princeton, Princeton University Press, 1987; Stanley Mellon, The Political Uses of History: A Study of Historians in the French Restoration, Stanford, Stanford University Press, 1958; Pierre Rosanvallon, Le Moment Guizot, Paris, Gallimard, 1985. 
détermineront la participation à la vie politique «active». Propriété agraire ou commerciale? Résidence rurale ou urbaine? Notabilité de famille ou «capacités" personnelles? Ces débats représentent une source privilégiée pour suivre l'apport du discours politique à la construction d'un imaginaire social, et le statut de la bourgeoisie ou de la classe moyenne au sein de celui-ci.

Deux séries de débats à la Chambre des députés, séparés de quelques années à peine, permettent de suivre l'évolution du langage social dans la vie publique ainsi que de mesurer l'impact de l'évènement. Décembre 1816 et janvier 1817 voient de vifs débats à la Chambre autour de la loi dite Lainé. Celle-ci, bien que socialement très restrictive - un électorat d'environ cent mille membres choisirait les députés au sein de quelque dix mille nantis - a le soutien des libéraux et attire les foudres des ultras. Ceux-ci font objection particulièrement à la provision selon laquelle les élections se dérouleraient dans le chef-lieu du département, entrainnant selon eux une surreprésentation des intérêts urbains et libéraux ${ }^{23}$. On évoque le danger des villes et des désordres typiques de la vie urbaine : foules révolutionnaires et armées impériales ne sont pas loin derrière. Mais c'est aussi l'identité des électeurs payant trois cents francs en taxes qui fait problème, et le débat voit ressurgir, dans le discours conservateur, le spectre du bourgeois dangereux parce que liminaire : trop riche pour être ignoré, pas assez pour être indépendant ou fiable.

Selon le leader ultra, le comte de Villèle, les électeurs à trois cents francs "ont une fortune commencée, une fortune à achever», et à ce titre sont par trop vulnérables aux pressions des administrations locales et du ministère (sousentendu : libéral) ${ }^{24}$. La classe moyenne - l'expression revient souvent - est, selon la droite, trop informe pour être capable d'indépendance. N'ayant acquis que partiellement fortune et éducation (un député parle de "demi-lumières»), les membres de ces groupes liminaires sont incapables de vertus publiques, comme le fait remarquer avec condescendance un autre député : "Je souscris de grand cœur à l'éloge des vertus domestiques de la classe moyenne. Mais ces vertus se trouvent aussi dans les autres classes; et d'ailleurs il ne s'agit pas ici de vertus domestiques mais de vertus publiques. ${ }^{25}$ Les classes moyennes, sous-éduquées et de ce fait incapables d'indépendance, se retrouvent mutilées dans le discours conservateur, reléguées au rang des autres groupes auxquels on refuse traditionnellement le vote pour la même raison, les femmes et les domestiques. Pour étayer leurs arguments, les conservateurs puisent dans la tradition du discours d'Ancien Régime sur le bourgeois comme créature en formation, trop instable et trop intéressé pour prendre part à la chose publique.

Face à ces objections, les libéraux n'offrent pas, dans ce contexte, de défense explicite ou éloquente de la bourgeoisie ou des classes moyennes. On

23. Guillaume de Bertier de Sauvigny, La Restauration, Paris, Flammarion, 1955, p. 146-147.

24. Archives Parlementaires de 1787 à 1860. Deuxième série, Paris, P. Dupont, 1862-1913, 127 volumes, volume XVII, p. 699-700.

25. Idem, p. 14-15. 
trouve bien ici et là un député pour faire l'éloge de la vitalité commerciale et des talents professionnels de «la classe moyenne», mais c'est là l'exception plutôt que la règle. Le chef de file des libéraux, Pierre-Paul Royer-Collard, se rabat sur une défense traditionnelle des vertus sociales de la loi, expliquant que «malgré son aspect démocratique [cette loi] n'efface point et ne peut point effacer ces grandes supériorités qui forment les aristocraties naturelles de la société et qui sont la force des gouvernements ${ }^{26}$. En 1816 encore, même un leader libéral peut invoquer le principe d'aristocratie (de talent, certes) dans une défense de l'extension du droit de vote.

C'est par rapport à ces débats de 1816-1817 que l'on peut saisir toute la nouveauté du langage politique de 1820, élaboré par les libéraux cette fois sous la pression d'une violente attaque réactionnaire. L'assassinat du duc de Berry a lieu alors même que le ministre libéral Élie Decazes, soumis à des pressions, collabore avec Villèle à une loi électorale plus restrictive. L'assassinat est l'occasion rêvée pour les conservateurs d'un raidissement qui commence avec le renvoi de Decazes (il a "glissé dans le sang", dit-on).

La presse est muselée, la police s'active, les ultras aussi ${ }^{27}$. En mai, tout est prêt pour le débat sur la loi dite du "double vote» : les collèges électoraux seraient sis dans les cantons ruraux et non au chef-lieu, et les électeurs les plus riches en milieu rural - des propriétaires terriens en toute probabilité conservateurs - voteraient une nouvelle fois au niveau du département pour remplir 172 sièges nouvellement créés. On imaginait facilement les conséquences pour la dominante idéologique de la Chambre. De fait, trois ans après l'adoption de la loi, il n'y restait que dix-huit députés libéraux ${ }^{28}$.

Cette fois-ci, battus en brèche par les circonstances politiques du moment, les libéraux tiennent un langage plus précis et plus audacieux quant aux intérêts sociaux et politiques qu'ils veulent incarner. L'opinion et la presse étant maintenant tenues au silence, la Chambre des députés demeure la seule arène publique ouverte à l'affrontement idéologique, d'où la passion qui informe maintenant les débats. Cette fois-ci les libéraux ne se retiennent plus d'invoquer les classes "moyennes», «industrieuses» voire "industrielles». Ainsi, le député Martin de Gray s’enflamme : «La prépondérance de la classe moyenne, c'est la vivante organisation de la France nouvelle; elle est nécessaire, parce que les forces physiques et morales lui appartiennent; parce que la balance des richesses et des idées a passé de son côté; elle est raisonnable et juste, parce que ses intérêts sont identifiés avec ceux de tout le corps du peuple, et qu'elle est particulièrement intéressée au maintien de l'ordre établi. ${ }^{29}$ On ne parle plus seulement de dispositions électorales, c'est la lutte

26. Idem, p. 698. 298.

27. François Furet, La Révolution de Turgot à Jules Ferry, 1770-1880, Paris, Hachette, 1988, p. 295-

28. Alan Spitzer, «Restoration Political Theory and the Debate over the Law of the Double Vote», dans Journal of Modern History, volume 55, $n^{\circ} 1$, mars 1983, p. 54-70.

29. Archives parlementaires..., ouv. cité, volume XXVII, p. 665. 
des classes qui prend forme rhétorique au cours de ces débats houleux : «Et pourquoi cet acharnement du parti aristocratique contre la classe industrielle et travaillante de la société?» demande Martin de Gray. "Pourquoi? C'est qu'il connaît le patriotisme du commerce et de l'industrie; il sait que dans tous les siècles et sur tous les points du globe, l'industrie et le commerce ont toujours fui le despotisme, et toujours suivi la liberté.» ${ }^{30}$ Acculés par la force de la réaction conservatrice, les députés libéraux n’hésitent pas à proclamer que la proposition de loi représente un coup de force de leurs adversaires aristocrates, qui ne cherchent rien de moins qu'à soustraire le vote à la classe moyenne industrielle et commerciale.

Laspect le plus frappant du discours libéral dans ces débats reste cependant le recours à l'histoire, presque complètement absent des débats de 1816-1817. À l'époque, les libéraux posaient en défenseurs de la Charte et de la monarchie, assurant leurs auditeurs que ces institutions représentaient l'essence atemporelle de la tradition politique nationale. En 1820, tout a changé : les libéraux dénoncent la loi du double vote en s'appuyant sur l'histoire, sur la tradition de domination et de violence qu'ils prêtent à la "race» de leurs adversaires.

Les passages les plus célèbres sont ceux que l'on doit à Royer-Collard, lesquels deviendront bientôt des morceaux d'anthologie. En 1817, il avait défendu la loi Lainé en s'appuyant sur une philosophie aristotélicienne du juste milieu. Trois ans plus tard, c'est en termes purement historiques qu'il célèbre l'avènement de la bourgeoisie. Dans sa plus célèbre oraison, celle du 20 mai 1820, il conclut un discours qui fait remonter au Moyen-âge le triomphe inéluctable des classes moyennes : «Les fleuves ne remontent pas vers leur source; les évènements accomplis ne retournent pas dans le néant. Une sanglante révolution avait changé la face de notre terre; sur les débris de la vieille société renversée avec violence, une société nouvelle s'était élevée, gouvernée par des hommes nouveaux avec des maximes nouvelles.» ${ }^{31}$

Il est vrai, cependant, que la Révolution pose un problème délicat pour Royer-Collard et ses collègues dans la formulation de leurs arguments. Comment se réclamer de l'héritage positif des évènements déclenchés en 1789 sans par là évoquer le spectre des violences populaires honnies par toute la classe politique de la Restauration? Comment se réclamer de la nécessité historique tout en récusant l'évènement régicide?

La solution consiste à présenter la révolution de 1789 non comme un point de départ mais comme un aboutissement. Dans un commentaire sur le régime issu de la Charte de 1814, Royer-Collard dépeint la Chambre des députés comme "la Nation en présence du trône et de l'aristocratie, la Nation dans cet état où elle a reçu la dénomination historique de commu-

30. Idem, p. 666.

31. Prosper de Barante, La vie politique de M. Royer-Collard, ses discours et ses écrits, Paris, Didier, 1863 ( $1^{\text {re }}$ édition 1861), 2 volumes, volume II, p. 30. 
nes». Et, ajoute-t-il, faisant écho aux défenseurs du tiers-état trente ans plus tôt, une fois retranchés monarchie et aristocratie, «ce qui reste de la Nation $[\ldots]$ est essentiellement homogène ${ }^{32}$. Le recours au mot "communes» dans ce contexte est particulièrement intéressant, étant donné la polyvalence du terme. Le mot renvoie de prime abord au modèle anglais, associant la Chambre des députés à la Chambre des communes britannique. Derrière cette association rassurante se profilent néanmoins des allusions plus provocatrices aux «communes» de 1789 (le tiers-état en rébellion), voire même à la très radicale commune de Paris. Mais surtout le terme "communes» sert à établir un lien organique entre le présent, le passé récent et controversé de 1789, et l'événement qui sert de pierre de touche historique aux libéraux de 1820 : "l'émancipation des communes", la création de villes franches par les rois de France à partir du XIIe siècle.

"La révolution a complété l'émancipation des communes» : la Révolution n'est nullement un point de départ, mais l'apogée d'un mouvement dont les origines s'enracinent profondément dans l'histoire de la nation, et dont l'aboutissement logique est la Charte de 1814. En remontant le temps historique, les libéraux réduisent la Révolution de cause majeure en conséquence nécessaire, et justifient du caractère inévitable d'un processus qui remonte non à trente ans mais à sept siècles. "Les premiers linéaments de la Charte furent tracés par Louis le Gros quand il affranchit les communes" explique Royer-Collard. «Alors commença sourdement, pour éclater au jour marqué dans le cours des siècles, la guerre légitime, mais terrible, du droit contre le privilège. $»^{33}$

L'histoire sert non seulement à lier les positions du groupe libéral au récit d'un avènement bourgeois pluriséculaire, mais aussi à évoquer le passé pour expliquer, en les noircissant, les agissements de leurs adversaires politiques. Certains intervenants libéraux dans le débat sur la loi du double vote n'hésitent pas à assimiler la proposition de loi à une résurrection du féodalisme. Pour Martin de Gray, la loi envisagée pourrait tout bonnement entraîner à sa suite le retour des abus les plus scandaleux de l'Ancien Régime : "Ainsi le sol de la France», conclut-il, "terre de liberté et de gloire serait insensiblement frappé de mainmorte et son peuple de servage!». Selon Martin de Gray, «le parti aristocratique" s'apprête à "conquérir une seconde fois les Gaules, de s'emparer de tout le territoire, des fabriques, des manufactures, et de toutes les richesses industrielles et commerciales de la France nouvelle $»^{34}$. Pour Royer-Collard, la loi du double vote ne vise rien de moins que la réintroduction du privilège au sein d'une société qui prétend l'avoir banni à jamais. Ce n'est pas seulement «la violation de la Charte, ce n'est pas seulement un coup d'État contre le gouvernement représentatif, c'est un

32. Idem, p. 20.

33. Idem, p. 16-17.

34. Archives parlementaires..., ouv. cité, volume XXVII, p. 667. 
coup d'État contre la société; c'est une révolution contre l'égalité; c'est la vraie contre-révolution ${ }^{35}$. L'argument historique des libéraux opère donc un brillant renversement rhétorique : ce sont en effet eux que l'on soupçonne toujours de fomenter la violence populaire, que l'on accuse de bafouer l'ordre et la légalité. Non, répliquent-ils, textes historiques à l'appui : les violents, les hors-la-loi, ce sont et ce furent toujours les aristocrates.

Le thème qui prend forme au sein de ces débats n'est autre que celui, promis à un brillant avenir, de la lutte des classes. C'est un motif que l'on retrouve, en dehors de l'enceinte de la Chambre, dans les écrits de ceux qui, au sein de la mouvance libérale ne participant pas aux débats, les historiens, journalistes et enseignants tels qu'Augustin Thierry, Français Guizot, ou François Mignet. Avant, mais surtout après la crise politique de 1820, ce sont eux qui élaborent dans leurs cours et leurs écrits le récit du triomphe de la bourgeoisie - triomphe bien moins économique que politique ${ }^{36}$. C'est ce récit dont, on le sait, s'inspirera Karl Marx mais que celui-ci transformera de fond en comble. Il importe donc de saisir dans toute sa spécificité la version française, autour de 1820, de la lutte des classes et de l'avènement bourgeois, d'autant plus que ce discours représente une exception majeure à la diabolisation habituelle de la bourgeoisie dans les représentations sociales françaises.

L'histoire de France telle que la racontent un Thierry ou un Guizot ne manque certes pas d'antécédents ou de modèles. La chronique de l'affrontement souvent violent entre monarchie et aristocratie remonte au moins aux querelles historiques du début du XVIII ${ }^{e}$ siècle autour des rôles respectifs des Gaulois, des Francs, et de la royauté. Le texte décisif du XVIII siècle demeure les Observations sur l'histoire de France (1765) de Gabriel Bonnot de Mably. Par sa critique aiguë de la noblesse, l'œuvre de Mably deviendra le texte historique de référence d'abord pour les philosophes et les révolutionnaires de la fin du siècle, et ensuite pour la génération des Royer-Collard et des Guizot (Guizot préside à sa réédition en 1823, pour laquelle il rédige une préface). C'est chez Mably que l'on trouve, de la manière la plus frappante, le récit des origines et des effets dévastateurs de l' «anarchie féodale». La noblesse française? Ce sont des guerriers sans scrupule qui, sous les Mérovingiens et les Capétiens, saisissent les terres et réduisent les Gaulois en esclavage. C'est à eux que les rois donnent des fiefs dans le vain espoir de les pacifier et de capter leur loyauté, sans jamais y parvenir. Ce sont ces hommes égoïstes et superbes qui font violence à leurs inférieurs et menacent leurs souverains même, tout en s'appropriant fort injustement le privilège héréditaire. C’est Mably également qui est à l'origine du récit de l'affranchissement des com- 
munes, et du rôle central du tiers-état comme l'allié naturel et fidèle de la monarchie. Contre la bourgeoisie, fidèle soutien des rois, s'élève une noblesse définie par l'égoösme, la violence, le principe de race et par un sang qui se voulait pur mais qui abreuvera les sillons d'un état nouveau.

Il est d'autant plus ironique que le concept explicite de la «lutte des classes" soit le fait d'un auteur aux sympathies royalistes et surtout aristocratiques, le comte de Montlosier. La publication de son De la monarchie française en 1814 a beau réjouir les conservateurs, les libéraux y trouvent aussi leur inspiration. Montlosier reprend en l'intensifiant le récit prérévolutionnaire des deux «races» françaises, Francs et Gaulois ${ }^{37}$. Pour réactionnaire que soit leur auteur, certains passages de l'ouvrage de Montlosier préfigurent la rhétorique d'un Thierry ou d'un Guizot, si ce n'est celle de Marx lui-même : "Nous allons voir s'élever au milieu de l'ancien état, un nouvel état; au milieu de l'ancien peuple un nouveau peuple [...]. Nous allons voir un état double, un peuple double, un ordre social double, marcher pendant longtemps parallèlement l'un à l'autre, s'attaquer ensuite, et se combattre avec acharnement. " ${ }^{38}$ Le récit pro-féodal du comte propose un modèle bientôt adapté aux besoins des libéraux : l'accent est mis sur l'affranchissement des communes, et Montlosier ravive le thème de l'affrontement entre monarchie et aristocratie, en y ajoutant cependant un acteur de poids : le «nouveau peuple» des villes. Surtout, le comte voit en la révolution l'aboutissement d'un processus pluriséculaire - et non, comme Barruel, le résultat d'un complot - et propose comme moteur de l'histoire française la lutte au sein de la nation entre deux «races» ou «classes» ennemies.

Des éléments d'une tradition historiographique remontant, via Montlosier, au dix-huitième siècle, se conjuguent donc avec les circonstances spécifiques de la Restauration - et surtout de la crise de 1820 - pour produire cette exception dans la tradition politico-culturelle française qu' est la glorification et l'héroïsation du bourgeois. Dès 1818, par exemple, Augustin Thierry, jeune disciple de Saint-Simon, en appelle à sa génération dans les pages du Censeur européen : «Ces sauveurs de nos arts, c'étaient nos pères; nous sommes les fils de ces serfs, de ces tributaires, que des conquérants dévoraient à merci; nous leur devons tout ce que nous sommes. À leur nom se rattachent des souvenirs de vertu et de gloire, mais ces souvenirs brillent peu, parce que l'histoire, qui devait les transmettre, était aux gages des ennemis de nos pères. ${ }^{39}$

L'historiographie des libéraux de 1820 donne donc sans ambages le beau rôle à la bourgeoisie : classe industrieuse, protectrice des "arts", soutien fidèle de la monarchie, rempart contre la violence aristocratique. Mais si les libéraux

37. Marie-France Piguet, Classe..., ouv. cité, p. 147-150; Shirley Gruner, «Political Historiography in Restoration France», dans History and Theory, n ${ }^{\circ}$ 8, 1969, p. 347-349.

38. Cité dans Marie-France Piguet, Classe..., ouv. cité, p. 149.

39. Cité dans Rulon Nephi Smithson, Augustin Thierry: Social and Political Consciousness in the Évolution of a Historical Method, Genève, Droz, 1973, p. 60. 
s'accordent pour donner le beau rôle à la bourgeoisie, ils divergent implicitement sur sa composition. Pour Augustin Thierry, la bourgeoisie s'apparente au tiers-état d'antan. Il chante les louanges du "patriotisme» et de "l'énergie" des "classes moyennes et classes populaires", et décrit les penchants "républicains» des «marchands et artisans» du XII e siècle. Guizot, au contraire, voit les villes médiévales se diviser rapidement entre une "bourgeoisie supérieure" et une "population ouvrière» encline aux vices et aux erreurs de la multitude. Thierry, en somme, reprend l'idéal révolutionnaire du peuple unifié dont la bourgeoisie serait désormais la tête de proue - un peuple défini par le travail et la productivité. Pour Guizot, il s'agit d'une nouvelle élite, mais non d'une élite industrielle. Voici comment il en décrit le noyau, dans son Histoire de la civilisation en France: "Ces juges, ces baillis, ces prévôts, ces sénéchaux, tous ces officiers du roi ou des grands suzerains, tous ces agents du pouvoir central dans l'ordre civil devinrent bientôt une classe nombreuse et puissante. Or, la plupart d'entre eux étaient des bourgeois; et leur nombre, leur pouvoir, tournaient au profit de la bourgeoisie et lui donnaient de jour en jour plus d'importance et d'extension. C'est peut-être là, de toutes les origines du tiers-état, celle qui a le plus contribué à lui faire conquérir la prépondérance sociale. ${ }^{40}$ C'est par ses fonctions administratives, par ses "capacités», et non par sa richesse seule que va triompher ce groupe ${ }^{41}$.

On voit donc comment l'idéal bourgeois ne s'impose pas en tant qu'hégémonie tranquille, mais dans la contestation, à travers les débats historiques et politiques des premières décennies du XIX ${ }^{\mathrm{e}}$ siècle. C'est un idéal qui restera fragile, selon nous, parce qu'il est bien plus lié aux aléas de la politique qu’à un inexorable triomphe du capitalisme. L'arrivée au pouvoir des champions de la bourgeoisie en 1830 coïncide, on le sait, avec l'apogée même de la «bourgeo-phobie». Comme l'a écrit François Furet, en s'identifiant à «la bourgeoisie» la monarchie de Louis-Philippe substitue «la cible fixe de l'État à une réalité mobile et insaisissable ${ }^{42}$.

On ne peut ici que suggérer certaines des raisons pour lesquelles le bourgeois devient le bouc émissaire sous la monarchie de Juillet. La résurgence des mouvements républicains et socialistes y est pour beaucoup, d'autant que nombre de ceux-ci s'inspirent d'un christianisme renaissant ${ }^{43}$. La distance que prend le régime de juillet par rapport à l'Église n'en rendra que plus facile la dénonciation du matérialisme et de l'égoïsme de la bourgeoisie naissante. À droite, les légitimistes et bonapartistes marginalisés ne se priveront pas non plus de se gausser du manque d'hérö̈sme et de la médiocrité spirituelle du régime de Juillet. Artistes, écrivains, et autres intellectuels sont eux aussi de

40. François Guizot, Histoire de la civilisation en France, Paris, Didier, 1979, 4 volumes, volume IV, p. 89-90.

41. Pierre Rosanvallon, Le Moment Guizot, ouv. cité, chapitre 5.

42. François Furet, La Révolution de Turgot à Jules Ferry, ouv. cité, p. 342.

43. Edward Berenson, Populist Religion and Leftwing Politics in France, 1830-1852, Princeton, Princeton University Press, 1984. 
la partie. Comme l'a suggéré Cesar Graña, l'hostilité de ces groupes envers "la bourgeoisie» reflète sans doute leur position commerciale, un marché galopant de l'écrit s'accompagnant d'une dépendance accrue des auteurs à son égard : la figure du bourgeois mesquin et bedonnant chez un Daumier ou un Balzac refléterait la peur et la colère qu'inspire la dépendance commerciale chez les artistes ${ }^{44}$. On pourrait illustrer la convergence de ces hostilités en mettant côte à côte trois classiques de la bourgeo-phobie parus tous les trois en 1846 : Le Peuple du républicain Jules Michelet; La Cousine Bette du légitimiste Balzac; et les Scènes de la vie de Bohème de Henry Murger.

La haine du bourgeois ne saurait ici encore s'expliquer simplement par un changement de régime économique : les historiens de l'économie s'accordent en effet pour conclure que la monarchie de Juillet ne vit pas plus de changement de fond dans les structures économiques que les décennies précédentes. Il faut attendre les années 1840 pour voir se développer les réseaux ferroviaires et les canaux, et les effets de l'intégration du marché national se feront surtout sentir après $1850{ }^{45}$. En dépit des figures emblématiques d'un Jacques Lafitte ou d'un Casimir Périer, la concentration capitaliste reste, en ces années, largement minoritaire, et la proportion des industriels dans les assemblées gouvernantes ne dépasse les $15 \%$ pas plus dans les années 1840 que dans les années $1820{ }^{46}$. Certes, les grandes villes, la capitale surtout, poursuivent la croissance spectaculaire entamée au siècle précédent, et le nombre de citadins de statut moyen s'accroît d'autant, mais on attend toujours une véritable synthèse sur la conscience de classe de ces groupes. Tout au plus sait-on qu'elle s'inscrit "en creux" dans la peur et la haine des classes "laborieuses et dangereuses $»^{47}$.

La haine du bourgeois telle qu'on l'appréhende à l'un de ses paroxysmes sous la monarchie de Juillet correspondrait donc bien moins à l'aversion envers un groupe social bien défini et conscient de soi, qu'à un ressentiment partagé par un large éventail de groupes envers les remous de la modernité (commercialisation, anonymat, croissance des villes), hostilité qui prend forme et trouve une expression surtout dans la sphère politique. C'est donc moins la norme bourgeoise qui serait à expliquer, - car en dehors du contexte politique de la Restauration celle-ci reste très difficile à saisir, - que la norme anti-bourgeoise : pourquoi s'acharner contre un groupe qui se revendique si peu?

C'est peut-être un autre idéal social que dessine, par la négative, le rejet

44. Cesar Graña, Bohemian versus Bourgeois: French Society and the French Man of Letters in the Nineteenth Century, New York, Basic Books, 1964, p. 37-42; voir aussi Jerrold Seigel, Bohemian Paris : Culture, Politics, and the Boundaries of Bourgeois Life, 1830-1930, New York, Penguin Books, 1987.

45. Richard Price, A Social History of Nineteenth-Century France, London, Holmes and Meier, 1987, p. 3-44; Christophe Charle, Histoire sociale de la France au XIXe siècle, Paris, Le Seuil, 1991, p. 34-38.

46. Pamela Pilbeam, The 1830 Revolution, Princeton, Princeton University Press, 1991.

47. Louis Chevalier, Classes laborieuses et classes dangereuses à Paris pendant la première moitié du XIXe siècle, Paris, Plon, 1958. 
du bourgeois. Si l'on en croit Hegel, l'antonyme du bourgeois ne serait pas l'aristocrate ou le prolétaire mais le citoyen. Dans la vision prémarxiste qui est celle du philosophe allemand, la caractéristique principale du bourgeois, c'est moins le type d'activité économique que le dévouement aux intérêts privés : la sphère domestique, d'une part, et l'intérêt (financier) particulier de l'autre. Le "matérialisme» du bourgeois se mesurerait à son inaptitude à accéder au domaine du collectif, du général, de la res publica. Le groupe que représente le bourgeois "en creux", l'idéal qu'il dessine par la négative, serait donc celui du service de l'État, incarnation historique - toujours selon Hegel - de l'universel et du sacré ${ }^{48}$. Du brillant administrateur monarchique au fonctionnaire républicain, des militaires impériaux aux "capacités» de la monarchie censitaire, le groupe normatif, celui qu'affirme en contrepoint l'image du bourgeois, ne serait-ce pas celui du serviteur de l'État?

Notre interrogation vise moins à apporter une réponse définitive à ces questions qu'à soulever un débat sur des thèmes qui dépassent le sujet précis de cet article. La réflexion précédente cherche à souligner la part du discours, et en particulier du discours politique, dans l'identification des groupes sociaux. Il va de soi que les définitions du social sont limitées par les conditions objectives du contexte - mais elles ne sont pas déterminées par celles-ci. Pour l'historien de l'imaginaire social, les questions intéressantes concernent "l'espace des possibilités entre la réalité sociale et ses représentations " ${ }^{49}$. C'est à partir de là que se posent un certain nombre de problèmes. Peut-on dire qu'un groupe, une classe, existe, s'il est identifié par ses détracteurs plutôt que ses membres? Comment intégrer une histoire de l'imaginaire social à l'histoire de la société? Comment tenir en équilibre l'histoire des données les plus immédiates de l'expérience sociale (l'accès aux ressources), l'histoire des pratiques sociales, et celle des représentations de la société? Du fait de sa fluidité et de son ambiguïté mêmes, l'histoire de la bourgeoisie constitue un chantier privilégié pour accéder à ces questions.

Sarah Maza est professeure à Northwestern University (Evanston/Chicago)

48. Charles Taylor, Hegel, Cambridge, Cambridge University Press, 1975 ; voir aussi la glose de Karl Marx, Kritik der Hegelschen Rechtsphilosophie, traduction française, Contribution à la critique de la philosophie du droit de Hegel, Paris, Aubier-Montaigne, 1971.

49. Dror Wahrman, Imagining the Middle Class: The Political Representation of Class in Britain, 1780-1840, Cambridge, Cambridge University Press, 1995, p. 14. 